Bundesgesundheitsbl 2021 · 64:102-103 https://doi.org/10.1007/s00103-020-03266-w Online publiziert: 7. Dezember 2020

(c) Springer-Verlag GmbH Deutschland, ein Teil von Springer Nature 2020

\title{
Einladung zur Fortbildungsveranstaltung für den Öffentlichen Gesundheitsdienst vom 24. bis 26. März 2021
}

Programm

Mittwoch, 24. März 2021

08.30-09.30 Uhr

Workshop (Anmeldung erforderlich): Aktueller Entwicklungsstand des Deutschen Elektronischen Melde- und Informationssystems für den Infektionsschutz

Michaela Diercke (RKI)

09.40-10.00 Uhr

Begrüßung

Prof. Dr. Dr. Andreas Hensel, Präsident des BfR

10.00-10.40 Uhr

Gesundheitliche Bewertung von E-Zigaretten

Dr. Elke Pieper (BfR)

10.40-10.50 Uhr Pause

10.50-11.30 Uhr

BfR Corona-Monitor - Risikowahrnehmung der Bevölkerung in Deutschland PD Dr. Gaby-Fleur Böl (BfR)

11.30-12.10 Uhr

Gentechnik in Lebens- und Futtermitteln

Hermann Broll (BfR)

12.10-13.00 Uhr Mittagspause

13.00-13.40 Uhr

Freund oder Feind: Toxin-bildende $B a$ cillus spp. als Pflanzenschutzmittel und Lebensmittelvergifter Dr. Hendrik Frentzel (BfR)
13.40-14.20 Uhr

Sektorübergreifende genombasierte Surveillance von Salmonellen in Deutschland

Dr. Michael Pietsch (RKI), Dr. Laura Uelze (BfR)

14.20-14.30 Uhr Pause

14.30-15.10 Uhr

Veganismus: Wahrnehmung der Bevölkerung und Darstellung in den Medien Dr. Mark Lohmann (BfR)

15.10-15.50 Uhr

Der Versorgungsstatus mit Vitaminen und Mineralstoffen bei veganer Ernährungsweise

Prof. Dr. Cornelia Weikert (BfR)

15.50-16.00 Uhr Pause

16.00-16.40 Uhr

Sicherheitsrelevante Aspekte bei Nahrungsergänzungsmitteln

Dr. Rainer Ziegenhagen (BfR)

Donnerstag, 25. März 2021

09.00-09.15 Uhr

Begrüßung

Prof. Lothar H. Wieler, Präsident des RKI

09.15-09.45 Uhr

Aktuelle Entwicklung der Corona-Pandemie in Deutschland

Dr. Osamah Hamouda (RKI)

09.45-10.15 Uhr

Die ÖGD-Kontaktstelle am RKI: Ziele, Aufgaben und Angebote

Dr. Ute Rexroth (RKI) 
10.15-10.45 Uhr

Impfung gegen Covid-19

Dr. Ole Wichmann (RKI)

10.45-11.00 Uhr Kaffeepause

11.00-11.30 Uhr

Kommt ein Virus geflogen - Meldungen von durch Stechmücken übertragenen Arbovirosen

Dr. Christina Frank (RKI)

11.30-12.00 Uhr

Bekämpfung von Stechmücken mit Vektorkompetenz

Dr. Anja Kehrer-Berger, Dr. Carola Kuhn (UBA)

12.00-12.30 Uhr

Nosokomiale lebensmittelbedingte Ausbrüche

Dr. Idesbald Boone (RKI)

12.30-13.15 Uhr Mittagspause

\subsection{5-13.45 Uhr}

Gesundheit 65+: Studie zur Gesundheit älterer Menschen in Deutschland

Dr. Judith Fuchs (RKI)

13.45-14.15 Uhr

Psychische Gesundheit der Erwachsenen in Deutschland: Ergebnisse des Berichts „Erkennen-Bewerten-Handeln“

Dr. Ulfert Hapke (RKI)

14.15-14.45 Uhr

Impfpflicht und Masernelimination

Dr. Anette Siedler (RKI)

14.45-15.00 Uhr Kaffeepause

15.00-15.30 Uhr

Neues von der KRINKO

Prof. Dr. Mardjan Arvand (RKI)

15.30-16.00 Uhr

Die globale Strategie der WHO zur Eliminierung von Virushepatitis $B$ und $C$ - wo steht Deutschland?

Dr. Ruth Zimmermann (RKI)
16.00-16.30 Uhr

Rahmenkonzept „Epidemisch bedeutsame Lagen - erkennen, bewerten und gemeinsam erfolgreich bewältigen “

Dr. Maria an der Heiden (RKI)

Freitag, 26. März 2021

09.00-09.15 Uhr

Begrüßung

Prof. Dr. Dirk Messner, Präsident des UBA

09.15-09.55 Uhr

5G aus der Sicht des Strahlenschutzes Dr. Blanka Pophof (Bundesamt für Strahlenschutz, BfS)

09.55-10.35 Uhr

Radon, die unterschätzte Gefahr - neue Regelungen für Wohnräume und Arbeitsplätze

Dr. Felix Heinzl (BfS)

10.35-10.45 Uhr Kaffeepause

10.45-11.25 Uhr

Hitzeaktionsplan für ältere Menschen in der Stadt Köln - Praxiserfahrungen des Gesundheitsamtes Köln

Dr. Hans-Guido Mücke (UBA),

Dr. Eleni Giannakidou-Jordan (Gesundheitsamt Köln)

11.25-12.05 Uhr

Corona-Viren in Innenräumen - Minimierung des Infektionsrisikos durch Lüftung

Dr. Wolfram Birmili (UBA)

12.05-12.50 Uhr Mittagspause

12.50-13.30 Uhr

Nachweise von SARS-CoV-2 im Abwasser - Gefahr oder Frühwarnsystem?

Dr. Hans-Christoph Selinka (UBA)

13.30-14.20 Uhr

Elementgehalte im häuslichen Trinkwasser

Dr. Christian Höra (UBA)

14.20-14.30 Uhr Kaffeepause
14.30-15.20 Uhr

Vorkommen multiresistenter Bakterien in der aquatischen Umwelt

Dr. Christina Förster (UBA)

15.20-16.00 Uhr

Neue Aspekte der Badegewässerhygiene Dr. Regine Szewzyk (UBA) 